\title{
Éditorial
}

\section{Traumatismes subis par les cyclistes et prévention des traumatismes}

\author{
I. B. Pless, C.M., M.D., FRCPC
}

En 1989, cette revue, bien avant que le terme "blessures » ne soit ajouté à son titre, publiait deux articles sur les traumatismes chez les enfants, et on m'avait demandé à cette occasion de rédiger un éditorial. J'avais alors choisi le titre « Deux aspects négligés de la prévention des traumatismes ${ }^{1}$, car j'avais l'impression qu'on omettait de souligner que les statistiques sur les traumatismes étaient insuffisantes (il n'y avait pas de données des services des urgences à l'époque) et qu'on ne mettait pas assez l'accent sur l'importance pour la santé publique des traumatismes chez les enfants. Par conséquent, il est intéressant de comparer les statistiques d'aujourd'hui avec celles d'il y a 25 ans et de constater les progrès accomplis.

Certains articles de ce numéro et du numéro précédent portent sur la sécurité des cyclistes en général et sur le port du casque en particulier. Bien qu'il s'agisse d'un objet quelque peu restreint, il peut servir d'indicateur sur la manière dont ce champ a évolué et sur ce qui reste à faire pour améliorer la recherche et la politique en la matière.

La documentation scientifique (et les médias) est encore truffée d'articles contre le port du casque de vélo et contre la législation sur le port du casque. Les arguments vont de "les casques sont inefficaces lorsqu'on se fait frapper par une voiture » (cela n'est vrai qu'en partie, car au moins un article précise que les casques offrent une certaine protection dans ces circonstances ${ }^{2}$ ) à " les casques envoient un mauvais signal, soit que rouler à vélo est dangereux " (c'est faux : rouler à vélo peut être dangereux) ${ }^{3}$. Qui plus est, il n'existe pas de données probantes solides indiquant que le port du casque rend le cyclisme plus dangereux, comme certains le suggèrent. Par exemple, un rapport étonnant ${ }^{4}$, dont les conclusions ont été par la suite discréditées ${ }^{5,6}$, suggérait que les casques encouragent les conducteurs à conduire plus près des cyclistes. Bon nombre d'articles anti-législation s'appuient sur une étude australienne ${ }^{7}$ selon laquelle légiférer entraînerait une diminution du nombre de cyclistes. Toutefois, ces articles ne font pas mention d'un grand nombre d'autres études, dont une étude à long terme sur les traumatismes crâniens chez les cyclistes menée par Olivier et collab. ${ }^{8}$, qui démontre de façon convaincante qu'il n'y a pas de déclin important après législation dans ce domaine. Les personnes qui s'opposent à la législation prétendent également qu'une diminution du nombre de cyclistes entraîne davantage d'obésité et autres conséquences néfastes pour la santé, mais aucun article ne contient de données probantes convaincantes à l'appui de cet argument.

$\mathrm{Ni}$ le rapport australien original ${ }^{7}$, ni aucune autre étude n'indiquent pendant combien de temps le nombre de cyclistes diminue (s'il y a lieu) après l'adoption d'une législation sur le port du casque. De plus, aucune étude n'a présenté de données physiologiques pour étayer l'affirmation selon laquelle de telles diminutions entraîneraient véritablement une baisse de la condition physique ou une hausse des

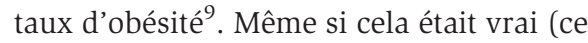
dont je doute, car la plupart des cyclistes ne pédalent pas assez longtemps ou assez vite pour brûler un grand nombre de calories), cela ne veut pas dire que la législation sur le port du casque soit dépourvue d'intérêt pour la santé publique. Les coûts - économiques, physiques et psychologiques - d'un traumatisme crânien grave sont importants. Dans ce contexte, l'importance d'une action préventive, notamment par la législation exigeant le port du casque, devient évidente. Comprenez-moi bien, je ne suggère pas que le problème de l'obésité chez les enfants canadiens ne soit pas important : il l'est. Toutefois, dans le contexte du débat à propos de la législation sur le port du casque, cela n'entre tout simplement pas dans le tableau.

Dans un jugement récent au Canada, on a accordé 3 millions de dollars à une famille dont l'enfant avait été grièvement blessé dans un accident de $\mathrm{ski}^{10}$. De plus, en Ontario, en une année, les frais médicaux pour les patients hospitalisés en raison d'un traumatisme cérébral se sont élevés à 120,7 millions de dollars ${ }^{11}$. Miller ${ }^{12,13}$, un économiste de la santé de premier plan aux États-Unis, et ses collaborateurs font invariablement état de l'excellent rapport coûts-avantages lié au port du casque. Dans l'une de leurs études, ils ont estimé que les coûts totaux des traumatismes cérébraux, qui comprennent les coûts attribuables aux soins médicaux, aux jours de travail perdus et aux changements dans la qualité de vie, étaient de 206 milliards de dollars (USD) pour 1,3 million de cas ${ }^{12}$. En ce qui concerne les traumatismes crâniens graves (de 4 à 6 sur l'échelle abrégée de gravité des traumatismes [AIS]), tous groupes d'âge confondus, le coût moyen par patient se chiffrait à plus d'un million de dollars (USD) et, pour les traumatismes très graves (AIS 6), à 3,3 millions de dollars (USD). Ne serait-ce que chez les enfants 
cyclistes victimes d'un traumatisme cérébral (grave et moins grave), le coût moyen s'élevait à 62000 \$ (USD) ${ }^{13}$.

Et il ne s'agit là que des coûts en dollars. Les coûts émotionnels pour les enfants et leur famille sont eux aussi stupéfiants. En revanche, les coûts liés aux cas d'obésité découlant directement de la réduction de la pratique du cyclisme - l'enjeu au cœur du débat dont nous traitons - ne sont pas connus, mais il est peu probable qu'ils s'apparentent aux coûts mentionnés dans les études sur les traumatismes crâniens. Pour obtenir des chiffres à peu près exacts, nous aurions besoin de savoir combien d'enfants ont cessé de faire du vélo en raison des lois sur le port du casque (jusqu'à maintenant, les données à cet égard sont incohérentes), combien de cyclistes sont devenus obèses du fait de l'abandon du vélo et, parmi ceux-ci, combien ont commencé à souffrir d'un diabète de type 2 ou d'une maladie cardiaque. Ensuite, nous aurions à estimer les coûts en dollars pour traiter ces maladies. À ma connaissance, personne n’a été en mesure de publier de telles estimations.

Qui plus est, sur le plan de la santé publique, il ne faut pas tenir compte uniquement des traumatismes crâniens graves. Mon expérience comme victime d'un « coup de portière » (voir la photo) m'a convaincu que si je n'avais pas porté de casque, ma blessure à la tête aurait été beaucoup plus grave. Ce type d'accident et d'autres incidents assez courants, par exemple les chutes provoquées par des nids-de-poule et les collisions avec des piétons imprudents, m'ont convaincu que le port du casque était essentiel, abstraction faite des accidents mettant en cause des voitures. Dans cette perspective, examinons ce que les articles de ce numéro de Maladies chroniques et blessures au Canada (MCBC) et du précédent peuvent apporter au débat.

Lindsay et Brussoni ${ }^{14}$ traitent des traumatismes subis par des patients pédiatriques au cours d'activités non motorisées sur roues, à savoir aussi bien en trottinette, en planche à roulettes et en patin à roues alignées qu'en vélo. Ils se sont servi de données tirées du Système canadien hospi- talier d'information et de recherche en prévention des traumatismes (SCHIRPT) ${ }^{15}$ pour générer un portrait de la façon dont les enfants peuvent se blesser lorsqu'ils utilisent ces moyens de transport « à roues ». La plupart des blessures sont attribuables au cyclisme, sont subies majoritairement par les garçons et sont surtout dues à des chutes. Jusqu'à maintenant, les résultats n’ont rien de remarquable. Cependant, environ $10 \%$ des patients ont présenté un traumatisme crânien, blessure particulièrement fréquente chez les cyclistes. Fait intéressant, les auteurs ont constaté que " les patients [...] dans une région où le port du casque était obligatoire étaient 2,12 fois plus susceptibles de déclarer porter un casque et 0,86 fois moins susceptibles de souffrir d'un traumatisme crânien, comparativement aux patients des régions où il n'y avait pas de législation sur le port du casque ${ }^{14,}$ p. ${ }^{79}$. J'adhère à leur conclusion, selon laquelle le nombre peu élevé de patients qui portaient un casque et un équipement de protection laisse penser que des interventions s'imposent toujours dans ce domaine ${ }^{14}$. Il est évident que ces interventions doivent inclure des efforts visant à convaincre les provinces où il n'y a pas de dispositions législatives rendant le port du casque obligatoire à remettre en question leur décision, comme cela s'est fait à l'égard de la ceinture de sécurité, aujourd'hui obligatoire dans toutes les provinces.

L'étude de Romanow et collab. ${ }^{16}$ publiée dans le numéro précédent se situe à un échelon supérieur sur l'échelle des données probantes, car elle repose sur une conception par cas-témoin. Les auteurs ont examiné l'effet de l'ajustement et de la position du casque de vélo sur le risque de blessure à la tête et au visage. Il n'est pas étonnant d'apprendre qu'ils ont observé qu'un casque mal ajusté ou mal placé pouvait faire augmenter jusqu'à six fois le risque de traumatisme crânien. Même si, d'un certain point de vue, cette conclusion ne fait que confirmer une évidence, elle demeure importante, car le mauvais ajustement et le mauvais positionnement du casque nuisent sans aucun doute à l'interprétation des résultats des premières études sur l'efficacité du casque protecteur. Sauf dans les cas où l'on a tenu compte de l'ajustement, ces études ont grandement sous-estimé l'efficacité des casques. C'est un peu comme si on avait examiné l'efficacité des ceintures de sécurité sans vérifier si elles étaient correctement ajustées ou attachées.

Enfin, un article de Parkin et collab. ${ }^{17}$, également dans le numéro précédent, a traité des attitudes et des croyances des parents au sujet du port du casque. La force de cette étude réside dans le fait qu'elle compare les provinces ayant légiféré dans ce domaine et celles ne l'ayant pas fait. Encore une fois, les résultats peuvent sembler prévisibles : les parents vivant dans une province ayant légiféré étaient plus favorables à la législation et à l'application de la loi que les parents vivant dans une province sans législation. Ce qui peut surprendre, toutefois, c'est que toutes les autres attitudes et croyances - les préoccupations à l'égard des accidents de vélo, les croyances au sujet de l'efficacité des casques, l'attitude à l'égard du port obligatoire du casque chez les enfants et, surtout, la croyance selon laquelle la législation entraîne une diminution du temps que consacrent les enfants au cyclisme - s'apparentent à celles observées dans les provinces «sans législation ».

Il est raisonnable de se demander ce qui vient en premier : les parents ayant une attitude raisonnable à l'égard de la sécurité à vélo ou la législation qui aide à modifier cette attitude? Il n'existe pas de données nous permettant de répondre à cette question pourtant importante. On dit souvent qu'une législation ne peut pas être adoptée sans un certain appui du public. Le seuil magique qu'il faut atteindre et l'origine des études à l'appui de cette vision demeurent un mystère. Du point de vue politique, il est beaucoup plus simple pour un législateur de faire la promotion d'une loi bénéficiant d'un vaste soutien du public que l'inverse. Toutefois, bon nombre de lois éclairées ont été adoptées sans un tel soutien. Il semble probable que si les autorités de santé publique jugent qu'un enjeu est suffisamment grave pour exiger une législation, les attitudes et les croyances à l'égard cet enjeu évolueront tôt ou tard dans la bonne direction. Inversement, certains parents peuvent s'interroger sur la pertinence à ce que 
leurs enfants portent un casque si leur province choisit de ne pas rendre le port de ce dernier obligatoire.

Oublions maintenant les chicanes législatives sur le port du casque et demandonsnous dans quelle mesure la sécurité à vélo a évolué au cours des 25 dernières années. Certaines statistiques sont encourageantes. Au cours de cette période, le nombre de blessures subies par les cyclistes a grandement diminué au Canada, sans que l'on sache vraiment à qui revient le mérite de cette diminution ${ }^{18}$. Les données de l'Institut canadien d'information sur la santé (ICIS) montrent que "bien que le nombre annuel d'hospitalisations en raison de blessures subies à vélo soit demeuré relativement stable entre 2001-2002 et 2009-2010, le nombre de traumatismes crâniens liés au cyclisme a diminué considérablement au cours de la même péri-

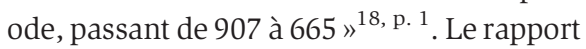
indique aussi que $78 \%$ des personnes hospitalisées en raison d'un traumatisme crânien ne portaient pas de casque protecteur au moment de l'accident ${ }^{18}$. Il demeure que les casques pourraient n'avoir qu'une légère incidence sur la mortalité à vélo. Le site Vehicular Cyclist $^{19}$ mentionne des données de Transports Canada de 1975 à 2003 qui, selon son interprétation, [traduction] " ne montrent aucun effet d'une utilisation accrue du casque protecteur parmi les cyclistes sur les tendances prédominantes de mortalité ». Cependant, il est évident lorsqu'on examine les données que le port du casque a augmenté et que les décès de piétons et de cyclistes ont diminué pendant cette période, de respectivement $42 \%$ et $39 \%$. L'article choisit d'attribuer ces diminutions aux [traduction] " campagnes de sécurité générale, comme celles où l'on fait subir des alcootests aux automobilistes ou celles où l'on assure la surveillance de la vitesse au moyen d'équipement radar ${ }^{19}{ }^{19}$ p. 1 , ce qui laisse entendre que l'utilisation du casque n'y a joué aucun rôle. Ces données ne signifient toutefois pas que les casques sont inefficaces, elles montrent peut-être simplement qu'un casque offre une protection bien relative contre des milliers de livres d'acier, et qu'une législation sans application est dénuée de sens.

En ce qui concerne les aspects négligés dont je faisais mention en 1989, avons- nous fait beaucoup ou peu de progrès? Chose certaine, nous disposons de meilleures données sur la morbidité attribuable à des traumatismes grâce au SCHIRPT et au Système national d'information sur les soins ambulatoires (SNISA), mais nous réagissons presque toujours aussi peu à ces résultats qu'en 1989. Même si plusieurs organismes ont vu le jour pour régler ce problème - le plus récent étant Parachute (http://parachutecanada.org/accueil), un regroupement de SécuriJeunes Canada, SAUVE-QUI-PENSE et de Communautés sécuritaires Canada - et que certaines provinces adoptent d'importantes initiatives en matière de prévention ${ }^{20,21}$, les mesures de santé publique relatives à la prévention des traumatismes au Canada demeurent insuffisantes.

En 1989, je concluais mon éditorial ainsi : « Toutefois, les meilleures statistiques ne sauraient suffire; il faudra un effort concerté dans le but d'aborder cette question de façon aussi dynamique que nous avons traité l'éradication des maladies transmissibles. Dans l'ensemble, la technologie et la [connaissance] du problème ne fait pas défaut; il reste à [...] constituer l'équivalent canadien de Injury in America. Avec

\section{FIGURE 1}

L'auteur, après avoir été victime d'un « coup de portière » alors qu'il se baladait à vélo dans une rue tranquille

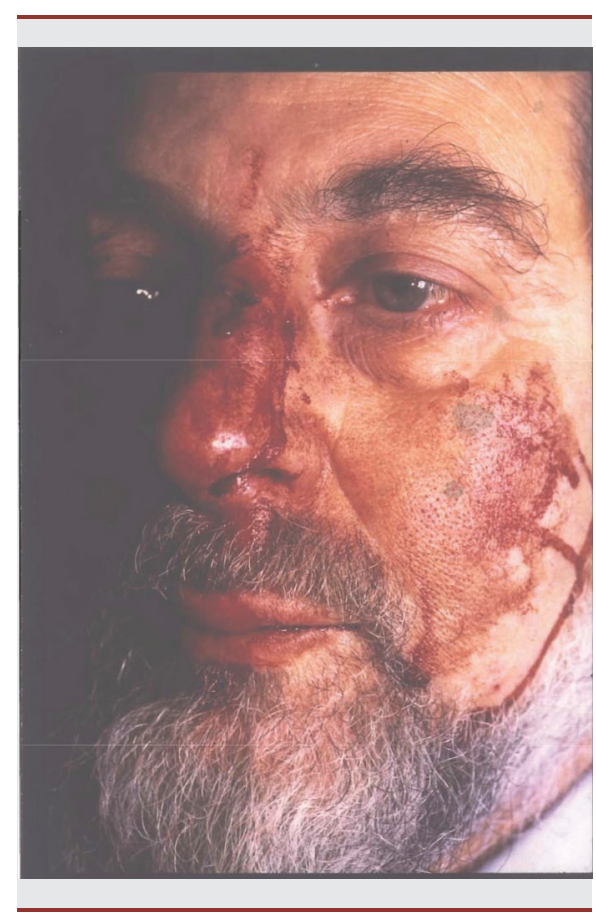

seulement un peu plus de ressources et un engagement solide, le Canada pourrait prendre les devants dans la course pour vaincre les traumatismes. Si nous n'empruntons pas cette voie, nous allons [...] croupir dans les ligues mineures pour une autre décennie, ou plus. »1, p. 2 . Aujourd'hui, je modifierais légèrement ce que j'ai écrit à l'époque, remplaçant le terme "décennie » par " quart de siècle ».

\section{Références}

1. Pless IB. Deux aspects négligés de la prévention des traumatismes. Maladies chroniques au Canada. 1989;10(1):2 .

2. Thompson DC, Rivara FP, Thompson RS. Effectiviness of bicycle safety helmets in preventing head injuries: a case-control study. JAMA. 1996;276(24):1968-73.

3. Chong S, Poulos R, Olivier J, Watson WL, Grzebieta R. Relative injury severity among vulnerable non-motorised road users: comparative analysis of injury arising from bicycle-motor vehicle and bicycle-pedestrian collisions. Accid Anal Prev. 2010;42(1): 290-6.

4. Walker I. Drivers overtaking bicyclists: objective data on the effects of riding position, helmet use, vehicle type and apparent gender. Accid Anal Prev. 2007; 39;417-25.

5. Olivier J, Walter SR. Bicycle helmet wearing is not associated with close motor vehicle passing: a re-analysis of Walker, 2007 [en ligne]. PLOS ONE. 2013;8(9):e75424 (doi:10 .1371/journal.pone.0075424)

6. Walter SR, Olivier J, Churches T, Grzebieta R. The impact of compulsory cycle helmet legislation on cyclist head injuries in New South Wales, Australia. Accid Anal Prev. 2011;43(6):2064-71.

7. Olivier J, Walter SR, Grzebieta RH. Long term bicycle head injury trends for New South Wales, Australia following mandatory helmet legislation. Accid Anal Prev. 2013;50:1128-34.

8. Robinson D.L. Head injuries and bicycle helmet laws. Accid Anal Prev. 1996;28:46375 . 
9. Pless IB. Are editors free from bias? The special case of letters to the editor. Inj Prev. 2006;12:353-4.

10. CanLII M.A.c. Stations de la Vallée de StSauveur inc., 2008 QCCS 240 [en ligne]. Ottawa (Ont.) : Institut canadien d'information juridique; 31 janv. 2008 [consultation le 11 mars 2013]. Consultable à la page : http://canlii.ca/t/1vlg5

11. Chen A, Bushmeneva K, Zagorski B, Colantonio A, Parsons D, Wodchis WP. Direct cost associated with acquired brain injury in Ontario. BMC Neurol. 2012;12:76.

12. Miller TR, Zaloshnja E, Hendrie DV. Cost of traumatic brain injury and the return on helmet investment in the United States. Dans: Jallo J, Loftus CM (dir.). Neurotrauma and critical care of the brain. New York : Thieme; 2009. p. 445-59.

13. Miller TR, Zaloshnja E, Lawrence BA, Crandall J, Ivarsson J, Finkelstein AE. Pedestrian and pedalcyclist injury costs in the United States by age and injury severity. Annu Proc Assoc Adv Automot Med. 2004;48:265-84.

14. Lindsay H, Brussoni M. Blessures et port du casque au cours d'activités non motorisées sur roues chez des patients pédiatriques. Maladies chroniques et blessures au Canada. 2013;34(2):79-87.

15. Mackenzie SG, Pless IB. CHIRPP: Canada's principal injury surveillance program. Inj Prev. 1999;5:208-21.

16. Romanow NR, Hagel BE, Williamson J, Rowe BH. Risque de blessures à la tête et au visage chez les cyclistes en relation avec l'ajustement du casque : une étude castémoins. Maladies chroniques et blessures au Canada. 2013;34(1):1-8.

17. Parkin PC, DeGroot J, Macpherson A, Fuselli P, Macarthur C. Attitudes et croyances des parents au sujet du port obligatoire du casque de vélo : comparaison entre provinces avec et sans législation. Maladies chroniques et blessures au Canada. 2013;34(1):9-13.
18. Institut canadien d'information sur la santé (ICIS). Registre des traumatismes : nombre d'hospitalisations au Canada en raison d'une blessure liée au cyclisme, 2009-2010 [Internet]. Ottawa (Ont.) : ICIS, juillet 2011 [consultation le 11 mars 2013]. PDF (140 Ko) téléchargeable à partir du lien : http:// www.cihi.ca/cihi-ext-portal/pdf/internet/info _cycling_injury_09-10_fr

19. The Vehicular Cyclist. Cyclist fatality trends in Canada: helmet effect undetectable in fatality trends [Internet]. 2007 [consultation le 11 mars 2013]. Consultable à la page : http://www.vehicularcyclist.com /fatals.html

20. Government of British Columbia. Injury prevention initiatives [Internet]. Government of British Columbia [consulté le 6 nov. 2013]. Consultable à la page : http://www .health.gov.bc.ca/prevention/injuryinitiatives .html

21. Alberta Centre for Injury Control \& Research. Injury Control Alberta [bulletin d'information en ligne]. Consultable à la page : http://acicr.ca/learn-and-network /newsletters 